\title{
Auf dem Weg zum MARS
}

\section{Jacques Huguenin}

Dr. oec., Leiter Projekt MARS, Bundesamt für Statistik, Neuchâtel

MARS steht für «Modules Ambulatoires des Relevés sur la Santé» und ist der Name des Projektes des Bundesamtes für Statistik (BFS) zum Aufbau der Gesundheitsversorgungsstatistik im ambulanten Bereich und zur Umsetzung von KVG Art. 22a. Im folgenden Artikel wird kurz über die Piloterhebungen und die erste produktive Erhebung des Teilprojektes «Strukturdaten Arztpraxen und ambulante Zentren» berichtet.

\section{Ergebnisse der ersten Piloterhebung}

Das Erhebungsteam des BFS ist mit den Ergebnissen aus der ersten Piloterhebung (Sommer 2015) sehr zufrieden. Teilgenommen haben 20 Einzelarztpraxen, Gruppenpraxen und ambulante Zentren verschiedener Ausrichtung. Dreiviertel waren deutschsprachig, ein Viertel französischsprachig. Die Erhebung wurde von der grossen Mehrheit der Pilotteilnehmer in dieser Form als durchführbar eingeschätzt. Vielen Dank auch an dieser Stelle den Teilnehmern.

Als grösste Schwierigkeiten erwähnten die Teilnehmer:

- Verständlichkeit der Fragen und der Fachbegriffe, insbesondere zum Thema Finanzen

- Hoher Zeitaufwand, weil die Information für einzelne Fragen schwierig zu beschaffen war. [Der Erfassungsaufwand betrug zwei Stunden (Median), Minimum 30 Minuten, Maximum vier Stunden.]

- Hoher Detaillierungsgrad der Fragen im Finanzund Personenkapitel (nicht ärztliches Personal)

Positiv wurden verzeichnet:

- Die Benutzerfreundlichkeit des elektronischen Fragebogens wurde mehrheitlich als gut bis sehr gut eingeschätzt.

- Die Schnittstellen zu FMH, MedReg, NewIndex und $\mathrm{RoKo}^{\odot}$. wurden erfolgreich benutzt und funktionierten zuverlässig.

- Rund 80\% der Pilotteilnehmer beurteilten Support und Dokumentationsmaterial als gut oder sehr gut.

- Die angewandte Fragebogentechnologie hat sich bewährt.

Aufgrund der Erkenntnisse aus der ersten Piloterhebung wird der elektronische Fragebogen zurzeit überarbeitet. Die wichtigsten Anpassungen sind dabei der Verzicht auf die Erhebung von Informationen über nicht ärztliche Gesundheitsfachpersonen auf Individualebene und die Optimierung der Benutzerführung. Neu werden nur noch neun Finanzzahlen zu aufsichts- rechtlichen Zwecken erhoben, die weiteren Finanzwerte dienen zu statistischen Zwecken. Insgesamt orientiert sich das Kapitel Finanzen stark an der RoKo ${ }^{\circ}$. Daneben werden zahlreiche Massnahmen umgesetzt, um den elektronischen Fragebogen zu verbessern und den Erfassungsaufwand zu reduzieren.

\section{Zweite Piloterhebung im Frühjahr 2016}

Die zweite Piloterhebung wird in Zusammenarbeit mit der FMH und der KKA im Frühjahr 2016 durchgeführt. Die im Rahmen der zweiten Piloterhebung erfassten Daten (Referenzjahr 2014) können von den teilnehmenden Arztpraxen für die erste produktive Erhebung im November 2016 - wie bei der elektronischen Steuererklärung - als Vorjahresdaten verwendet werden.

\section{Erste produktive Erhebung November 2016}

Die erste produktive Erhebung wird im November 2016 durchgeführt werden. Befragt werden alle Arztpraxen und ambulanten Zentren der Schweiz. Dies, weil die Erhebung gemäss KVG Art. 22a und 23 für aufsichtsrechtliche und statistische Zwecke gleichzeitig erfolgt. Die Verarbeitung und Verwendung der Daten erfolgt für die beiden Zwecke strikte getrennt. Personendaten werden bereits im elektronischen Fragebogen anonymisiert, also bevor sie zur getrennten Weiterverarbeitung ins BFS gelangen [1].

\section{Wie können Sie sich vorbereiten?}

Für die Erhebung im November 2016 ist es wichtig, dass der Finanzabschluss 2015 vorliegt. Im Weiteren werden Sie durch die Schnittstellen zu FMH / MedReg / NewIndex $/ \mathrm{RoKo}^{\odot}$ unterstützt. Und ein lohnender Tipp bereits vorweg: Lesen Sie die Anleitung «Das Wichtigste in Kürze». Dies in Analogie zur Aufforderung: Lesen Sie die Packungsbeilage ... 